\title{
Application of Adaptive Neuro-Fuzzy Inference System in High Strength Concrete
}

\author{
Behnam Vakhshouri \\ $\mathrm{PhD}$ candidate \\ CBIR \\ University of Technology Sydney
}

\author{
Shami Nejadi \\ Senior Lecturer \\ CBIR \\ University of Technology Sydney
}

\begin{abstract}
Abstract: Adaptive Neuro-Fuzzy Inference System is growing to predict nonlinear behaviour of construction materials. However due to wide variety of parameters in this type of artificial intelligent machine, selecting the proper optimization methods together with the best fitting membership functions strongly affect the accuracy of prediction. In this study the nonlinear relation between splitting tensile strength and modulus of elasticity with compressive strength of high strength concrete is modelled and the effect of different effective parameters of Adaptive Neuro-Fuzzy Inference System is investigated on these models.

To specify the best arrangements of parameters in the System to utilize in high strength concrete properties, different combinations of optimization methods and membership functions in the Sugeno system have been applied on more than 300 previously conducted experimental datasets. Both the grid partition and sub-clustering methods have been applied to models and compared to get the best combination of parameters.
\end{abstract}

\section{Keywords}

ANFIS, High strength concrete, Compressive strength, Splitting tensile strength, Modulus of Elasticity

\section{INTRODUCTION}

High Strength Concrete (HSC) is an especial type of concrete to use in modern construction projects mostly in tall buildings and towers to reduce the cumulative weight of the structure. Application of HSC for tall buildings began in the 1970s, primarily in the U.S.A. Use of HSC continues to spread, particularly in the Far East and Middle East [1]. The definition of high-strength concrete has changed over the years [2]. In the 1950 s, concrete with a compressive strength (CS) of $5000 \mathrm{psi}$ (34 MPa) was considered HSC [1]. American Concrete Institute (ACI), committee 363-92 selected 6000 psi (41 MPa) as a lower strength limit for HSC [2]. Nowadays, due to advances in concrete technology, it is possible to reach high levels of CS and new classes of strength are introduced in recent classifications in different design codes. While HSC in ACI $363.2 \mathrm{R}$ has a specified CS of 8000 psi $(55 \mathrm{MPa})$ or greater, ACI 211.4R-08 specified CS equal to or less than 6000 psi (41 MPa) for normal strength concrete [3].

Accurate prediction of mechanical characteristics of hardened concrete is almost the basic stage in design and evaluation of reinforced concrete structures. In conjunction with the traditional methods including destructive and non-destructive tests and empirical relations to predict mechanical properties of concrete, artificial intelligent based modelling methods have been applied to simulate non-linear and complex behaviour of various properties of construction materials in the recent years.
For ANFIS based soft sensor models, when estimation/prediction accuracy is concerned, it is assumed that both the data used to train the model and the testing data to make estimations are free of errors (Klein and Rosin, 1999), but rarely a dataset is without error (Jassar et al., 2009). Several studies have investigated the effect of data errors on the outputs of computer based models. Bansel et al. (1993) studied the effect of errors in test data on predictions made by neural network and linear regression models [4].

\section{MATERIALS}

Almost all mechanical properties of concrete could be estimated by the most important structural property of concrete, CS. Selected mechanical properties of HSC in this study include Splitting Tensile Strength (STS), Modulus of Elasticity (MOE) and CS that are essential in all type of design and evaluation of HSC structures. This study considers MOE and STS as input and CS as output in ANFIS model. More than 100 sets of experimental studies in the last 15 years has been collected from Giaccio and Zerbino(1998) [5], Jin-Kuen and Sang-Hun (1999) [6], Shannag (2000) [7], Ajdukiewicz and Kliszczewicz (2002) [8], Jin-Keun et al. (2004) [9], Bissonnette et al. (2007) [10], Almeida et al. (2008) [11], Pablo (2008) [12], Yin, J. et al. (2010) [13], K. M. Ng et al. (2010) [14], Ozbay et al. (2011) [15], Parra et al. (2011) [16], Das and Chatterjee (2012) [17], Ranaivomanana et al. (2013) [18].

To include wide range of experimental data in the model, lower and upper limit of CS for HSC is selected $400 \mathrm{MPa}$ and 1000 $\mathrm{MPa}$ respectively. Table 1 , represents the range of collected experimental mechanical properties for HSC in this study.

Table 1. Statistical values of mechanical properties of HSC in this study

\begin{tabular}{|c|c|c|c|}
\hline $\begin{array}{c}\text { Mechanical } \\
\text { property }\end{array}$ & $\begin{array}{l}\text { Min. } \\
\text { value }\end{array}$ & $\begin{array}{l}\text { Max. } \\
\text { value }\end{array}$ & Average \\
\hline MOE (GPa) & 21.3 & 49.9 & 38.37 \\
\hline STS (MPa) & 2.28 & 7.4 & 4.33 \\
\hline $\mathrm{CS} \quad(\mathrm{MPa})$ & 40.4 & 98.8 & 57.01 \\
\hline
\end{tabular}

Artificial intelligent model in ANFIS trains and compiles data to establish a fuzzy logic between input and output values. To validate the accuracy of the logic, it should be tested by some other data from the experiments. To develop this model in ANFIS, 240 datasets from the total of 305 datasets (79\%) of HSC mechanical properties, are considered as training data and the remaining $21 \%$ as testing the data. To have similar distribution of data in training and testing process, data is classified in 6 equivalent groups of CS as indicated in table 21 . Then testing data have been selected based on percentage weight of each group. 
Table 2. Classification of data and number of selected testing data in each class

\begin{tabular}{|c|c|c|c|c|c|c|}
\hline CS (MPa) & $\mathbf{4 0 - 5 0}$ & $\mathbf{5 0 - 6 0}$ & $\mathbf{6 0 - 7 0}$ & $\mathbf{7 0 - 8 0}$ & $\mathbf{8 0 - 9 0}$ & $\mathbf{9 0 - 1 0 0}$ \\
\hline Percentage in all data & 48 & 24 & 6 & 10 & 8 & 4 \\
\hline Number of testing data & 10 & 5 & 1 & 2 & 2 & 1 \\
\hline
\end{tabular}

\section{ARTIFICIAL INTELLIGENT BASED MODELS AND ANDIS}

To implement the fuzzy logic technique to a real application, the following three steps are required:

1. Fuzzification: converts classical data or crisp data into fuzzy data or Membership Functions (MFs)

2. Fuzzy Inference process: combines MFs with the control rules to derive the fuzzy output

3. Defuzzification: returns a defuzzified value out, of a MF positioned at associated variable value $\mathrm{x}$ using one of several defuzzification strategies, according to the argument type.

Fuzzy systems, Artificial Neural Network (ANN), adaptive network-based inference, neuro-fuzzy and genetic fuzzy systems are types of new generation of simulation and modelling methods called artificial intelligent-based modelling methods that is applicable in all fields of science. In the field of civil and material engineering, it has been applied to simulate non-linear and complex behaviour for various properties of construction materials in recent years [21].

Fuzzy systems, is particularly useful in the engineering applications where classical approaches fail or they are too complicated to be used. ANFIS is a class of adaptive networks which has the advantages of ANN and linguistic interpretability of Fuzzy Inference Systems (FIS) $[2,19]$.

Application of ANFIS was first proposed by Jang (1993) [20] used ANFIS to predict the CS of high performance conventional concrete from fresh concrete properties. Sadrmomtazi et al. (2013) [21] applied ANFIS analysis to study the relation between CS of lightweight concrete and mixing proportion.

\subsection{Variables in ANFIS model}

ANFIS utilizes different variables including normalization method, trial step quantity and various data classification methods to achieve the minimum error between predicted values and real data. Number and type of MF, type of output MF, optimization method (hybrid or back propagation) and the number of epochs are five important adjustments in ANFIS to reach the most effective model with minimum errors. This paper studies the effect of these adjustments and their subdivisions in different combinations to develop new ANFIS models and compare the results. For this purpose, all possible combinations of these adjustments are applied to unique sets of training and testing data.

\subsection{Fuzzy rule-based inference system}

Mamdani and Tagaki-Sugeno fuzzy architectures are two basic and well-known fuzzy rule-based inference systems in artificial intelligent based modelling [22] that are mainly different in the way to generate crisp output from the fuzzy inputs [23] and linear or constant type of output MF [22]. Sugeno FIS uses weighted average to compute the crisp output, while Mamdani FIS uses the technique of defuzzification of a fuzzy output [23, 24].
Mamdani FIS has interpretable and intuitive nature of the rule base and is widely used in decision support application and studying human input [22], while Sugeno FIS is more compact and computationally efficient representation and is well suited to mathematical analysis.

Sugeno FIS lends itself to the use of optimization and adaptive techniques for constructing fuzzy models and works well with linear techniques (e.g., Proportional-IntegralDerivative (PID) control). These adaptive techniques can be used to customize the MFs so that the fuzzy system best models the data. It also has guaranteed continuity of the output surface [25]. Therefore considering these abilities and advantages of Sugeno FIS especially in mathematical and design systems, all models in this study are developed based on the Sugeno FIS system.

\subsection{Optimization methods}

ANFIS applies two optimization methods for FIS training. First method is Hybrid Optimization Method (HOM) that is default method in ANFIS and uses combination of least squares and back-propagation to optimize the predicted relations. The second method is known as back-propa (BackPropagation) Method (BPM). ANFIS performs the training operation on the given real input and output data based on error tolerance to create a training stopping criterion, which is related to the error size. When the training data error remains within this tolerance, the training will stop. Both type of optimization methods are utilized in this study.

\subsection{Membership functions}

Generally, fuzzification involves two processes; deriving the MFs for input and output variables and representing them with linguistic variables. Fuzzy algorithm categorises the information entering a system and assigns values that represent the degree of membership or degree of truth in those categories. In fuzzy logic, degree of truth as an extension of valuation is presented by MFs associated with terms that appear in the antecedents or consequent of rules.

By applying MF in fuzzy system that is similar to generalization of the indicator function in classical sets, a fuzzy system allows members to have a smooth boundary rather than classical sets.

In practice, MFs can have multiple different types. Fig. 1 shows the mathematical model and distribution shape of 8 types of the MF including trimf, trapmf, gbellmf, gaussmf, gauss $2 \mathrm{mf}$, pimf, dsigmf and psigmf that have been used in this study. In addition there are other types of MF like S-curve, and Z- shape that could be used in practical studies of fuzzy systems. The MF choice is the subjective aspect of fuzzy logic; it allows the desired values to be interpreted appropriately. The exact type and shape of MF to apply in each actual application depends on the purpose of the study and parameters defining the uncertainty distribution function. Relations between input uncertainty and MFs may be estimated analytically [26]. For those systems that need significant dynamic variation in a short period of time, a triangular or trapezoidal waveform should be utilized. For 
those system that need very high control accuracy, a Gaussian or S-curve waveform may be selected. In the case of concrete material properties [21] used the bell-shaped MF in the ANFIS models.

\subsection{Output membership function}

As mentioned before, there is just a constant MF available in Mamdani FIS, but Sugeno architecture uses either linear or constant type of output for MF. Therefore the Sugeno FIS uses optimization techniques to find best parameters to fit data instead of trying to do it heuristically. The present study applies and compares both types of linear and constant output MFs in combination with various optimization methods and different numbers of epochs.

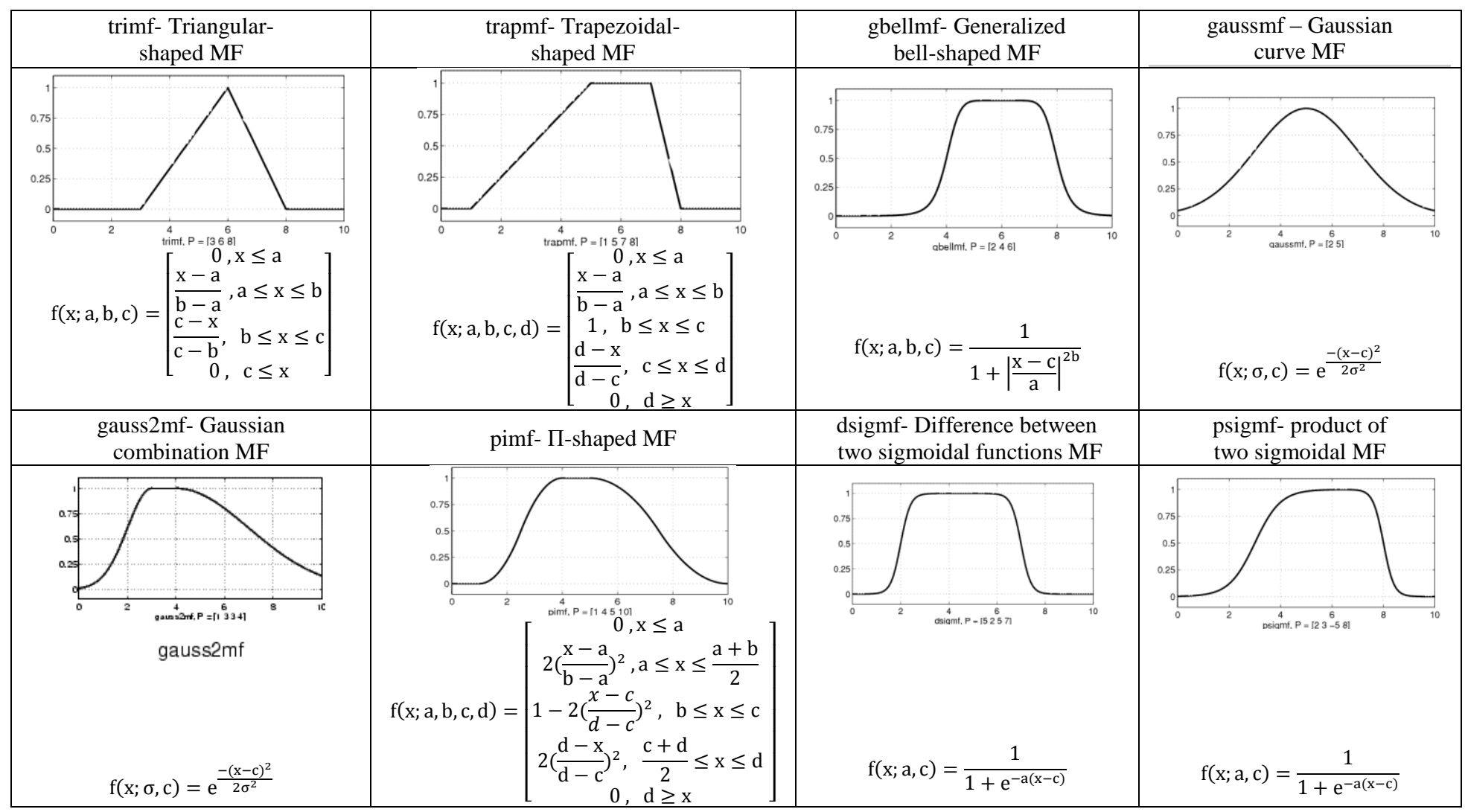

Fig. 1 - Shape and mathematical equations of utilized MF types

\section{DEVELOPING ANFIS MODELS IN MECHANICAL PROPERTIES OF HSC}

Mechanical properties of HSC from different experimental studies since 1998 have been collected. More than three hundred datasets including STS, MOE and corresponding CS at the age of 28 days are implemented in ANFIS neuro-fuzzy system. CS is known as the most important characteristic of concrete particularly in HSC and generally most of $E=f\left(f_{c}^{\prime}\right) \quad, \quad f_{t}=g\left(f_{c}^{\prime}\right)$ therefor: Fuzzy logic

This study implements Sugeno FIS architecture because of its ability to give more reliable results in design type and mathematical datasets respect to Mamdani FIS. As typically illustrated in Fig. 2, this adaptive neuro-fuuzy version is constructed based on fuzzy "if-then" governing rules and trains a set of applied input variables to produce a single predicted output [27]. mechanical properties of concrete like flexural strength, direct tensile strength, STS and MOE are stated in terms of CS in design codes, scientific and engineering references. In other words, CS is a common characteristic between mechanical properties of concrete. Consequently according to if-then rule in Eq. (1), STS and MOE are considered as input data to give CS as output.

\section{if $\left[E\left(f_{c}^{\prime}\right) \& f_{t}\left(f_{c}^{\prime}\right)\right]$ then $f_{c}^{\prime} \quad$ Eq. (1)}

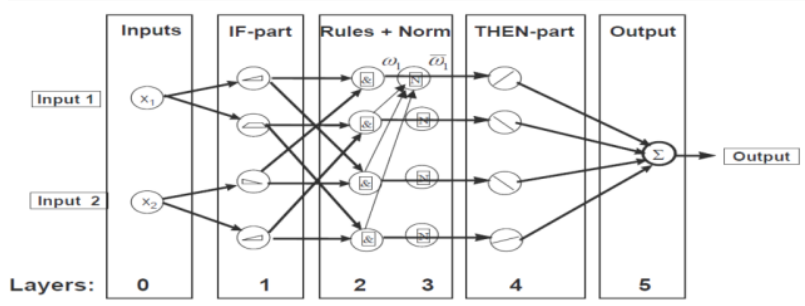

Fig. 2: Fuzzy "if-then" governing rules to predict single output from input variables 
Both hybrid and back-propagation neural network method of optimization in six layers are integrated in the model to remember experimental data pertaining to MOE and STS versus 28 days CS relationship of experimental investigations since 1998. The entire dataset of mechanical properties of HSC in this study are 305 datasets that are referred to 240 datasets (79\%) as training data and 65 datasets $(21 \%)$ as testing data.

After successful training, further testing data (not included in training data) are applied to see how the ANFIS behaves for known data. The testing data, evaluates the generalization capability of the FIS at each epoch. In both training and testing, ANFIS shows the error size which reflects the how compatible the mapping function is [28]. The error size computes the discrepancy between the network's actual output and a desired output.
By utilizing BPM, some ANFIS models couldn't run the training process in six layers, so another successful optimization was done by five layers.

To evaluate the effect of reduced layers in accuracy of training and testing process, successfully trained models in six layers were trained again in five layers. The difference between the results is not significant and comparison between different combinations of the effecting parameters is acceptable. Although applying more layers together with adjustment between the quantity of linear and non-linear parameters with number of training data pairs, can give better training results (less error), however, it doesn't guarantee the same behaviour to improve testing results. Figs. 3(a,b) show developed Sugeno ANFIS network models in 5 and 6 layers respectively.

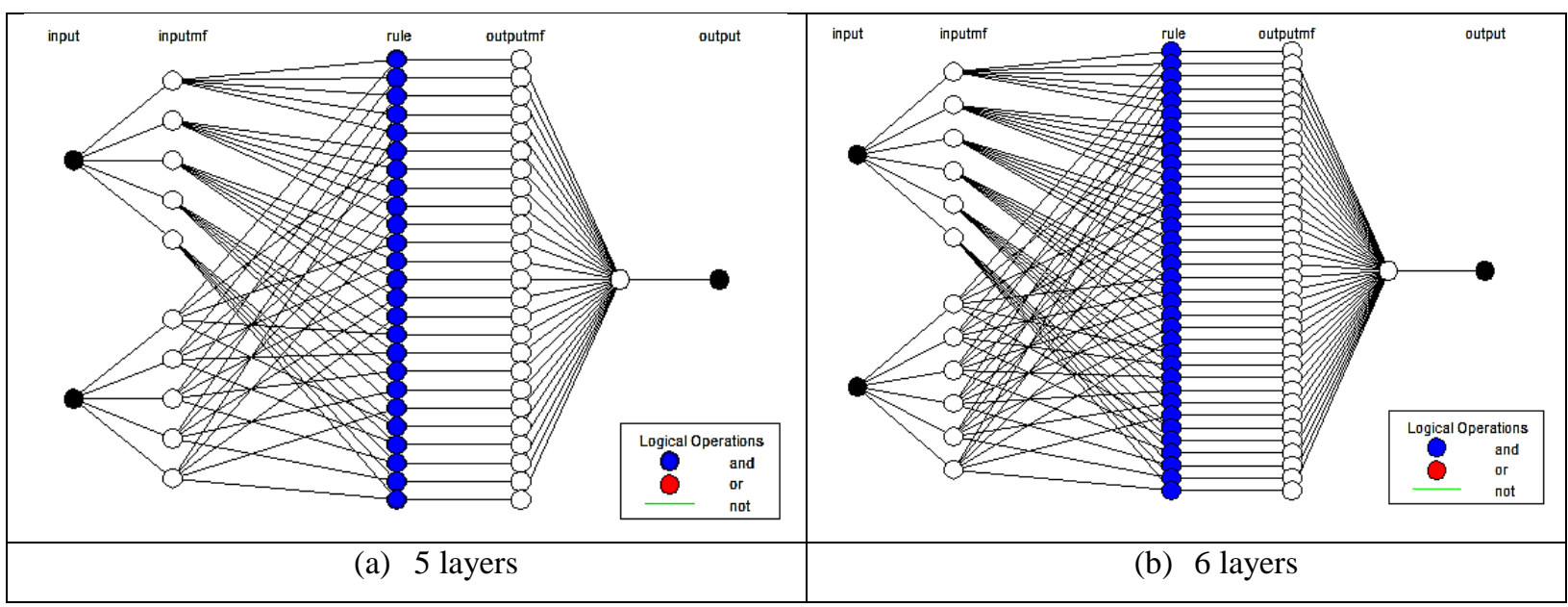

Figs. 3: (a) 5 layered and (b) 6 layered structure of Sugeno FIS network model of input and output data

To achieve comprehensive results from developed models in ANFIS, all possible combinations of input and output variables together with various optimization methods are studied. Table 3, presents the combinations of the parameters included in this study. Corresponding results for testing and training error size are given to evaluate the compatibility of predicted values and to assess divergence between the actual output data and a desired output.
According to table 3 , the training and testing errors are strongly influenced by applying different optimization methods and output and input MFs on the model. Figs. 4 (a, b) graphically compare the error values in training and testing processes resulting from the hybrid and backpro optimization methods by applying linear or constant output type for different types of MF in input data.

Table 3: Combinations of parameters in Sugeno FIS models of mechanical properties of HSC

\begin{tabular}{|c|c|c|c|c|c|c|c|c|c|c|}
\hline \multirow{2}{*}{$\begin{array}{l}\text { Optimization } \\
\text { method }\end{array}$} & \multirow{2}{*}{$\begin{array}{c}\text { Output } \\
\text { type }\end{array}$} & \multirow{2}{*}{ Error } & \multicolumn{8}{|c|}{ MF TYPE } \\
\hline & & & trimf & trapmf & gbelmf & gaussmf & gauss $2 \mathrm{mf}$ & pimf & dsigmf & psigmf \\
\hline \multirow{4}{*}{ Hybrid } & \multirow{2}{*}{ Constant } & train error & 5.8521 & 6.1999 & 4.2608 & 3.9331 & 5.453 & 5.9261 & 5.502 & 5.3688 \\
\hline & & test error & 9.3378 & 9.1953 & 136.8276 & 39.7901 & 8.8778 & 8.6221 & 8.6544 & 8.6217 \\
\hline & \multirow{2}{*}{ Linear } & train error & 1.8296 & 2.3103 & 0.38313 & 1.611 & 0.29654 & 1.2687 & 0.1589 & 0.15887 \\
\hline & & test error & 96.1268 & 24.4759 & 42.6255 & 1928.961 & 565.8644 & 23.1614 & 190.8436 & 191.236 \\
\hline \multirow{4}{*}{$\begin{array}{c}\text { Back } \\
\text { propagation }\end{array}$} & \multirow{2}{*}{ Constant } & train error & 15.52 & 13.7877 & 10.9798 & 13.8687 & 15.6731 & 7.2205 & 13.59 & 15.4113 \\
\hline & & test error & 14.3483 & 14.7646 & 9.9606 & 15.4846 & 16.1461 & 12.9785 & 12.19 & 14.3285 \\
\hline & \multirow{2}{*}{ Linear } & train error & 6.0937 & 7.0143 & 6.5929 & 7.8125 & 8.2939 & 7.0429 & 5.6228 & 5.3414 \\
\hline & & test error & 10.893 & 12.5256 & 9.5679 & 10.7854 & 14.8134 & 14.3438 & 12.3457 & 13.6315 \\
\hline
\end{tabular}


It should be noted that unique values of actual input and output data from the investigated mechanical properties of HSC are implemented in all combinations, so comparing the results is pretty reasonable and reliable. According to Fig. 4(a), the best compatible mapping function in training is achieved from the HOM with linear output type and psigmf input MF. While utilizing the BPM with constant output value and gauss $2 \mathrm{mf}$ input MF gives the biggest value of deviation. In general, hybrid-linear provides more compatible mapping than hybrid-constant, backpro-linear and backpro-constant method which are in lower levels of compatibility in training process.

According to Fig. 4(b), the best compatible mapping function in testing is achieved from HOM with constant output type and pimf input MF. While, utilizing the hybrid method with linear output type and gaussmf input MF gives the largest

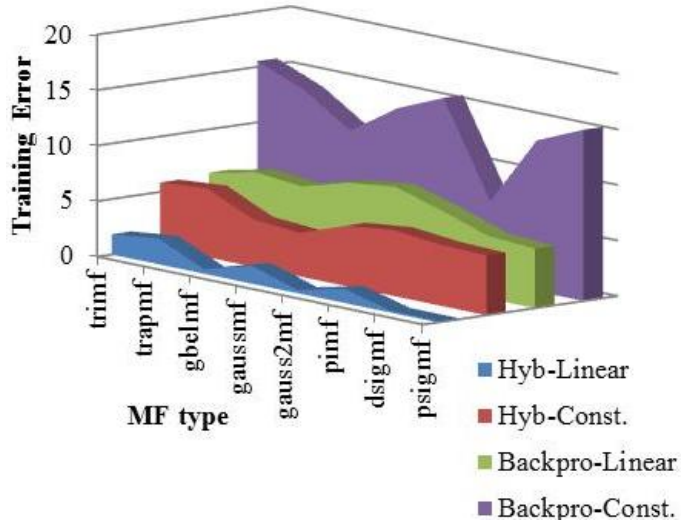

value of divergence. Generally, hybrid-constant, backprolinear and backpro-constant provide similar levels of compatibility in mapping; conversely, hybrid-constant is completely different and gives the worst predictions of testing. Figs. 5 (a, b, c, d) shows the most and least compatibility results for training and testing of developed models in ANFIS as mentioned previously.

As general criterion in ANFIS models, the less testing error gives more reliable predictions, but according to table 3 , while training and testing errors are not in the same level. It could be mentioned that models with testing error close or up to two times value of training error, are reasonable to be considered for output values.

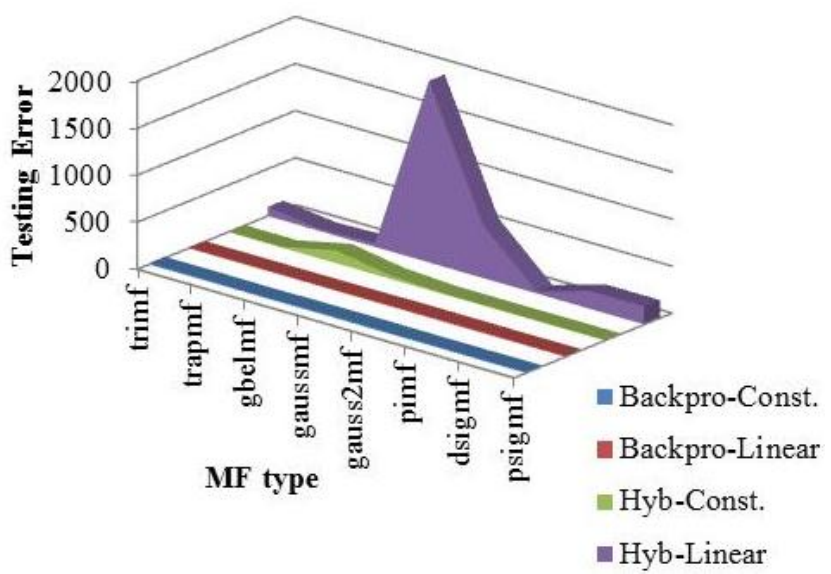

Fig. 4: Training and testing error resultant from HOM and BPM by various input and output MFs

As shown for a sample in Figs. 5, generally BPM needs much more epochs to reach the error criteria and to stop the training

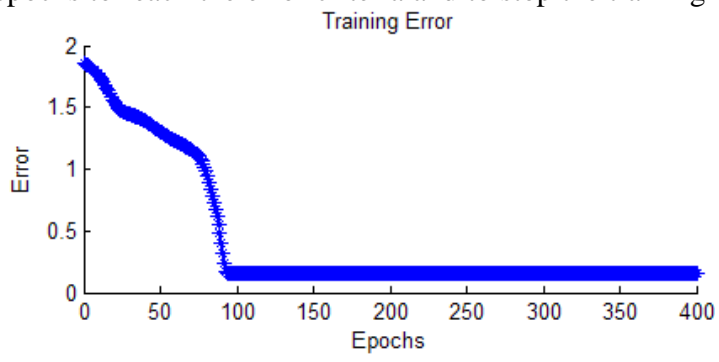

Training with hybrid-linear-sigmf

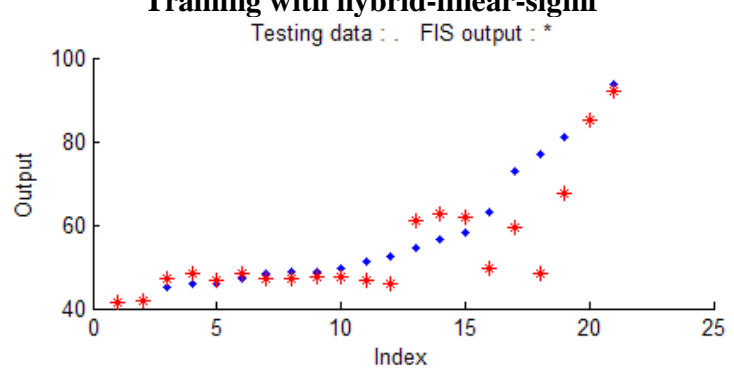

Testing with hybrid-constant-pimf process, but it doesn't necessarily ensure the better predictions.

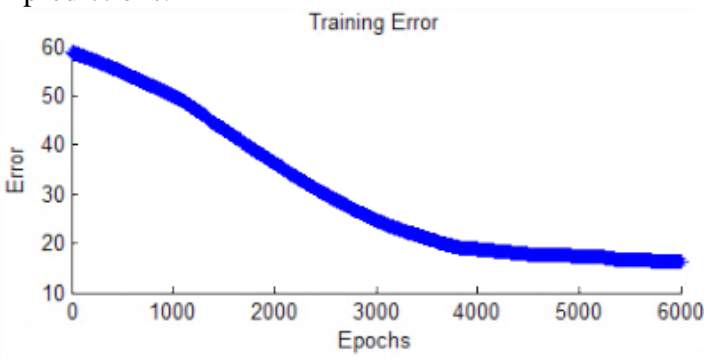

Training with backpro-constant-gauss2mf

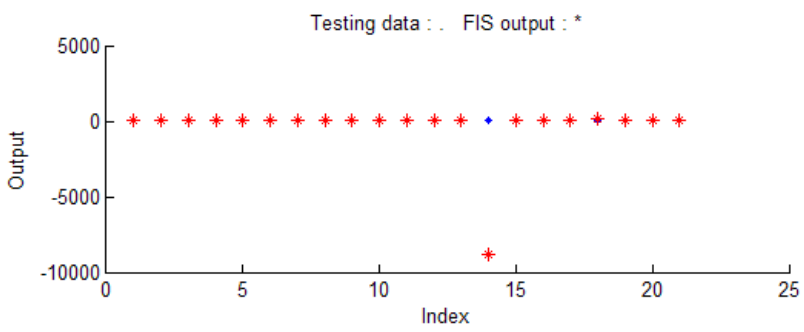

Testing with hybrid-linear-gaussmf

Fig.5: The most and least compatible results in training and testing by different combinations of parameters

Figs. 6 (a, b, c, d) graphically compare the error values in the individual training and testing process of each optimization method utilizing different MFs.
In most of the combinations, the difference between training and testing error is not considerable. But due to large amount of divergence in testing of constant-gbellmf, constantgaussmf, linear-gaussmf and linear-gauss $2 \mathrm{mf}$ of 
HOM, these combinations are not suitable to use in HSC studies. However in the case of BPM, the results are more reasonable than hybrid method as the amount of divergence is much less than hybrid method. Mostly, the testing error is less than two times value of training error. Just in psigmf and disgmf MFs with linear output type, testing error slightly exceeds the two times of training error.

Consequently, combinations in which the error size of training and testing is close (testing error equal to or up to two times value of training error) are recommended to be used in studying mechanical properties of HSC. But evidently, depending on the application purpose, data range and number of datasets, allowable error criteria may change.

To compare the two optimization methods of Sugeno FIS, the average values of training and testing errors resulted from different MFSs are considered. Figs. 7 (a, b) show the training and testing errors in all combinations and mean value of training and testing errors in different combinations of optimization methods and output type respectively.

According to Fig. 7 (b), considering testing error, developed models with BPM in both linear and constant types of output, give more compatible mapping between exact data and model desired output. In other side, both models of hybrid method provide predictions out of generally accepted range.

The ratio of testing error to training error is completely different in BPM and HOM. While the results in the first method are reasonable and the ratio is less than 2, especially in the constant output type in which the ratio is approximately 1 , corresponding ratios in hybrid methods are in high ranges.

Hybrid Opt. Constatn output type

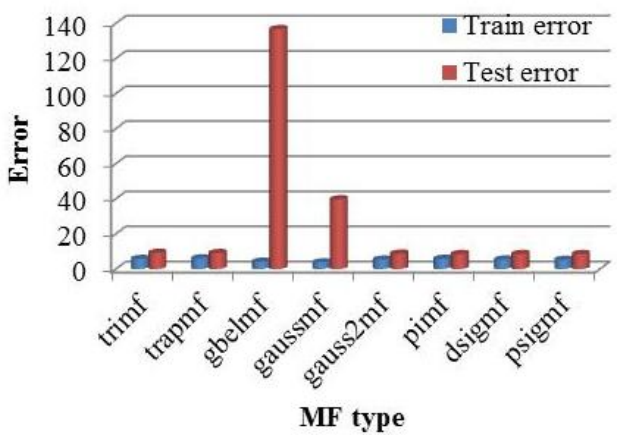

Backpropagation Opt. Constant output type

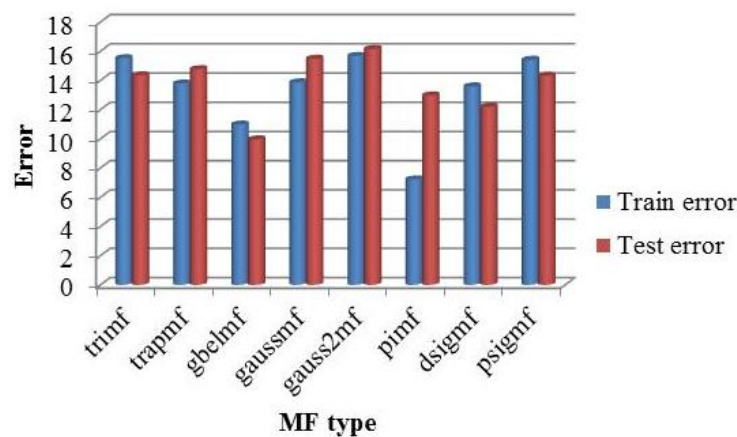

According to table 3 , the main problem dealing with the obtained results from almost all ANFIS models is the high values of testing and training errors. Since all the models have been developed based on the same input and output data, comparing the results even with high range of errors may be reasonable. But logically, a model in which the divergence between predicted output and actual data tends to zero is more acceptable.

All the FIS models in table 3 are generated by Grid Partition Method (GPM). This method divides the data space into rectangular subspaces using axis-paralleled partition based on pre-defined number of MFs and their types in each dimension (Neshat et al., 2011) [27]. Subtractive Clustering Method (SCM) is another method of generating FIS model that implements each data point as a potential cluster center and calculates the potential for each data point based on the density of adjacent data points. Then data point with maximum potential is selected as the first cluster center and the potential of data points close to the first cluster center is destroyed. Then data points with the highest remaining potential as the next cluster center and the potential of data points near the new cluster (Wei et al., 2007) [29].

To reduce the size of errors and evaluating the uncertainties resultant from high range of errors in GPM, the ANFIS models have been developed to SCM. The key factors of SCM are as indicated in table 4. Similar to GPM, different combinations of key factors in SCM have been applied to models and compared.

Hybrid Opt. Linear output type

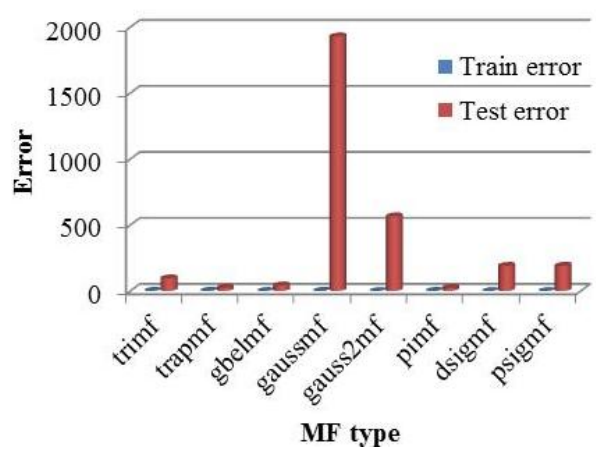

Backpropagation Opt. Linear output type

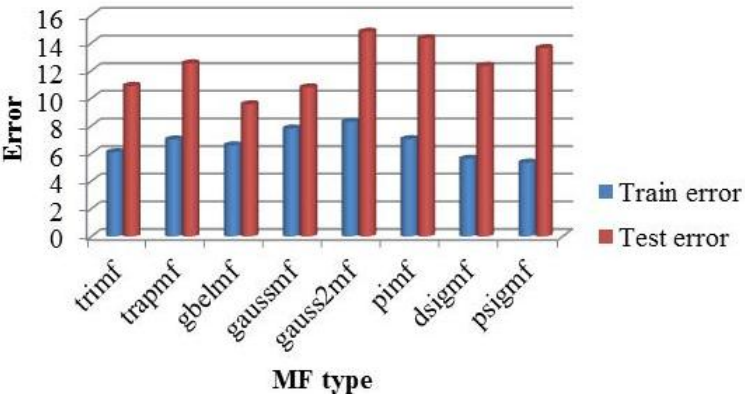

Fig. 6: Resultant training and testing errors from hybrid and backpro method with various input and output MFs 


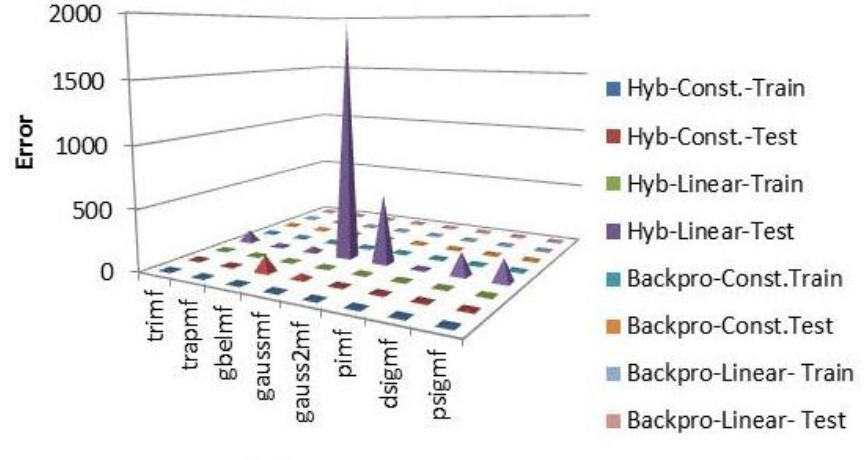

MF type

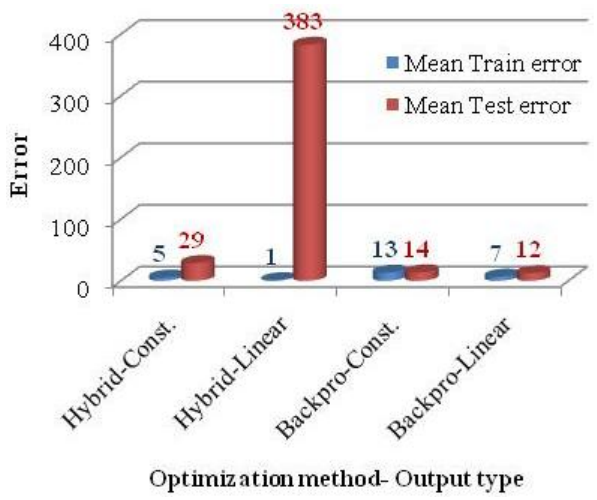

(b)

Fig. 7: a) Comparative diagram of individual exact training and testing errors in different MFs and b) comparative diagram between mean values of all MFs in two types of optimization

Default values of IR, SF, AR and RR in ANFIS are 0.5, 1.25, 0.5 and 0.15 respectively. Different combinations of these factors give completely different training and testing errors. To evaluate the effect of each factor in training and testing of implemented data for HSC properties, keeping constant the values of the 3 corresponding factors, various values of each individual factor were studied. For example, to evaluate the effect of IR, different models were developed with changing value of IR with constant values of SF, AR and RR. Figs. 8 (a, $\mathrm{b}, \mathrm{c}$ and d) show the effect of each factor in training and testing errors of ANFIS models.

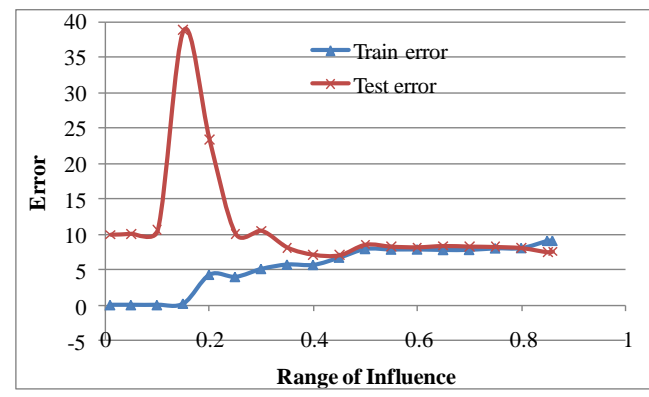

(a)

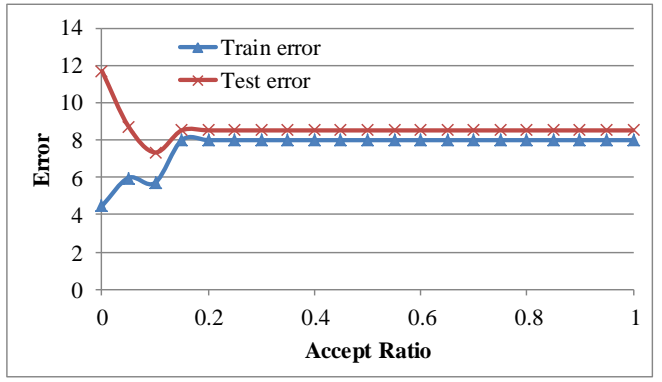

(c)
Table 4: Key factors of Subtractive Clustering method to generate FIS models

\begin{tabular}{|c|l|}
\hline Factor & \multicolumn{1}{c|}{ Function } \\
\hline $\begin{array}{c}\text { Influential } \\
\text { Radius (IR) }\end{array}$ & Directly affect the clustering result \\
\hline $\begin{array}{c}\text { Squash factor } \\
\text { (SF) }\end{array}$ & $\begin{array}{l}\text { Used to multiple the given radii values to } \\
\text { squash the potential of outlying points to } \\
\text { be considered as part of that cluster }\end{array}$ \\
\hline $\begin{array}{c}\text { Accept Ratio } \\
\text { (AR) }\end{array}$ & $\begin{array}{l}\text { Sets the potential as a fraction of the } \\
\text { potential of the first cluster center and } \\
\text { above which a data point will be accepted } \\
\text { as a cluster center }\end{array}$ \\
\hline $\begin{array}{c}\text { Reject Ratio } \\
\text { (RR) }\end{array}$ & $\begin{array}{l}\text { Sets the potential as a fraction of the first } \\
\text { cluster center and below which a data } \\
\text { point will be rejected as a cluster center. }\end{array}$ \\
\hline
\end{tabular}

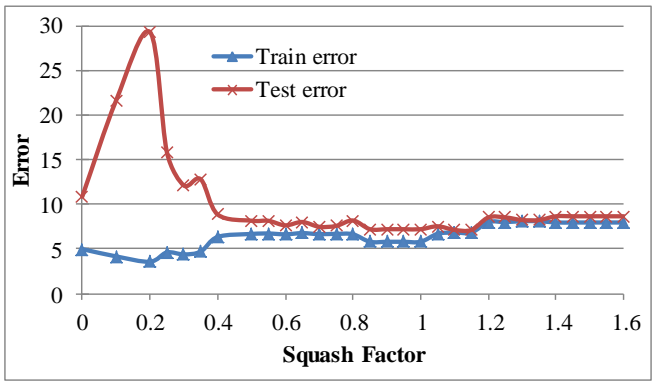

(b)

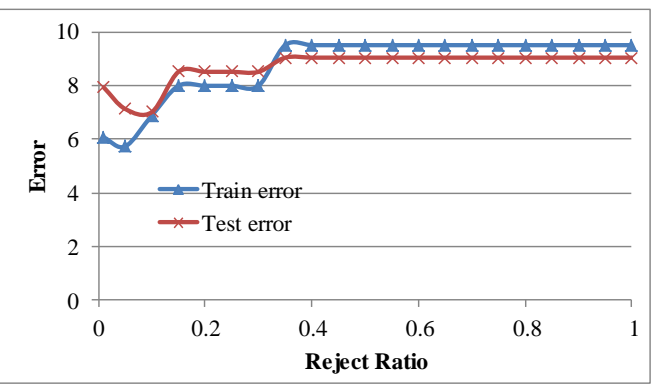

(d)

Figs. 8: Effect of a) IR, b) SF, c) AR and d) RR in compatibility of developed models with actual data 
According to Figs. 8 (a, b, c and d), RI and SF have similar effects on developed models, while AR and RR behave similarly. Considering default values of ANFIS for SF, AR and $\mathrm{RR}$, the error range of training and testing has sever fluctuations for RI $<0.5$ and tend to be stable after $I R>0.5$. The least error (best compatibility) for testing could be achieved by IR around 0.5 , however the best compatibility of training error is issued from IR about zero. Other diagrams could give similar conclusions for training and testing error size of models. Advantage of these individual diagrams of factors is to enable designer to choose best combinations for different applications and goals. However, to have comprehensive comparison database, different combinations of these factors are included in models and the results are presented in table 5. Fig. 9 compares the results from table 5 .

According to Fig.9, the difference in error sizes from various combinations of coefficients in table 5 is not considerable and majority of errors are less than 10 . In combination 3 with equal coefficients, BPM gives the maximum error size both in testing and training process and according to Fig. 8a, gives a high level of testing error in combination 13. While hybrid method gives the maximum testing error size in combination 10 with default values of ANFIS.

\section{RECOMMENDATIONS AND CONCLUSIONS}

Adaptive Neuro-Fuzzy Inference System (ANFIS) models was developed utilizing more than 300 datasets of previously conducted experiments on mechanical properties of HSC to investigated the predict CS from STS and MOE.
Comparing the Mamdani and Sugeno FIS architectures in ANFIS, the Sugeno FIS was selected to apply on models. Both methods of grid partition and sub-clustering were applied to generate the models. All possible arrangements of different types of optimization methods, input and output MFs in grid partition method have been considered. Individual effect of each factor in sub-clustering method investigated and 30 combinations of these factors to achieve the best compatibility between actual and predicted values of output data have been performed.

Default values in ANFIS for both grid partition and subclustering don't guarantee the accuracy of predicted output values.

In general, hybrid-linear provides more compatible mapping than hybrid-constant, backpro-linear and backpro-constant method which are in lower levels of compatibility in training process.

- Considering mean value of error of all combinations in training and testing, both models of hybrid method provide predictions out of generally accepted range.

RI and SF have similar effects on developed models, while AR and RR behave similarly.

The combinations in which the error size of testing is close to training error, (up to twice value) are recommended to be used in studying mechanical properties of HSC. However, regarding the study goal, parameters include, range and number of datasets, allowable error criteria may change.

Table 5: Different combinations of SCM factors to achieve the best compatibility between actual and predicated output data

\begin{tabular}{|c|c|c|c|c|c|c|c|c|}
\hline & \multicolumn{2}{|c|}{ sub clustering coefficents } & \multicolumn{2}{c|}{ Hybrid method } & \multicolumn{2}{c|}{ Backpro method } \\
\cline { 2 - 11 } & RI & SF & AR & RR & Train error & Test error & Train error & Test error \\
\hline $\mathbf{1}$ & 0.1 & 0.1 & 0.1 & 0.1 & 0.079057 & 10.7871 & 1.3441 & 11.139 \\
\hline $\mathbf{2}$ & 0.2 & 0.2 & 0.2 & 0.2 & 0.37837 & 65.63 & 171.9405 & 125.4327 \\
\hline $\mathbf{3}$ & 0.3 & 0.3 & 0.3 & 0.3 & 2.385 & 38.717 & 31513 & 15787 \\
\hline $\mathbf{4}$ & 0.5 & 0.5 & 0.5 & 0.5 & 6.8151 & 8.5082 & 6.5166 & 8.3276 \\
\hline $\mathbf{5}$ & 0.50 & 1.25 & 0.50 & 0.15 & 7.9993 & 8.5222 & 8.2224 & 8.13 \\
\hline $\mathbf{6}$ & 0.40 & 1.25 & 0.50 & 0.15 & 5.7485 & 59.4575 & 6.143 & 24.47 \\
\hline $\mathbf{7}$ & 0.30 & 1.25 & 0.50 & 0.15 & 5.1829 & 10.4982 & 5.4437 & 6.9088 \\
\hline $\mathbf{8}$ & 0.20 & 1.25 & 0.50 & 0.15 & 4.362 & 23.3443 & 4.6183 & 8.8283 \\
\hline $\mathbf{9}$ & 0.10 & 1.25 & 0.50 & 0.15 & 0.079057 & 10.5453 & 1.049 & 12.7126 \\
\hline $\mathbf{1 0}$ & 0.50 & 1.25 & 0.50 & 0.10 & 6.891 & 594575 & 7.5618 & 8.4724 \\
\hline $\mathbf{1 1}$ & 0.40 & 1.25 & 0.50 & 0.10 & 5.3585 & 7.4856 & 5.4594 & 16.0323 \\
\hline $\mathbf{1 2}$ & 0.30 & 1.25 & 0.50 & 0.10 & 5.1829 & 10.4982 & 5.3895 & 7.1057 \\
\hline $\mathbf{1 3}$ & 0.20 & 1.25 & 0.50 & 0.10 & 2.8404 & 32.2165 & 5719.1888 & 5719.0301 \\
\hline $\mathbf{1 4}$ & 0.10 & 1.25 & 0.50 & 0.10 & 0.079057 & 10.5453 & 0.98461 & 12.7644 \\
\hline $\mathbf{1 5}$ & 0.50 & 0.85 & 0.50 & 0.15 & 5.7935 & 7.2018 & 6.6061 & 8.9627 \\
\hline $\mathbf{1 6}$ & 0.50 & 0.90 & 0.50 & 0.15 & 5.7935 & 7.2018 & 6.6061 & 8.9627 \\
\hline $\mathbf{1 7}$ & 0.4 & 1 & 0.4 & 0.1 & 4.6893 & 8.8516 & 5.1066 & 7.9642 \\
\hline $\mathbf{1 8}$ & 0.3 & 1 & 0.3 & 0.05 & 3.7816 & 21.6565 & 27.0156 & 35.2149 \\
\hline $\mathbf{1 9}$ & 0.2 & 0.9 & 0.2 & 0.05 & 0.96361 & 26.8212 & 1.1062 & 19.8803 \\
\hline $\mathbf{2 0}$ & 0.6 & 1.15 & 0.1 & 0.1 & 6.8567 & 7.7563 & 7.0954 & 9.1198 \\
\hline $\mathbf{2 1}$ & 0.8 & 0.8 & 0.2 & 0.2 & 7.1996 & 7.4159 & 8.1147 & 8.262 \\
\hline $\mathbf{2 2}$ & 0.2 & 0.2 & 0.8 & 0.8 & 7.2225 & 7.3259 & 6.4234 & 7.1726 \\
\hline $\mathbf{2 3}$ & 0.50 & 1.25 & 0.40 & 0.15 & 7.9993 & 8.5222 & 8.1967 & 8.2423 \\
\hline $\mathbf{2 4}$ & 0.50 & 1.25 & 0.30 & 0.15 & 7.9993 & 8.5222 & 8.1967 & 8.2423 \\
\hline $\mathbf{2 5}$ & 0.50 & 1.25 & 0.20 & 0.15 & 7.9993 & 8.5222 & 8.1967 & 8.2423 \\
\hline $\mathbf{2 6}$ & 0.50 & 1.25 & 0.10 & 0.15 & 5.7141 & 7.3396 & 6.7377 & 8.8877 \\
\hline $\mathbf{2 7}$ & 0.50 & 1.15 & 0.50 & 0.15 & 6.8289 & 7.1216 & 7.5115 & 8.1572 \\
\hline $\mathbf{2 8}$ & 0.50 & 1.05 & 0.50 & 0.15 & 6.6835 & 7.5287 & 7.5284 & 8.7259 \\
\hline $\mathbf{2 9}$ & 0.50 & 1.00 & 0.50 & 0.15 & 5.7935 & 7.2018 & 6.5912 & 9.0539 \\
\hline $\mathbf{3 0}$ & 0.50 & 0.95 & 0.50 & 0.15 & 5.7935 & 7.2018 & 6.5842 & 9.1069 \\
\hline
\end{tabular}




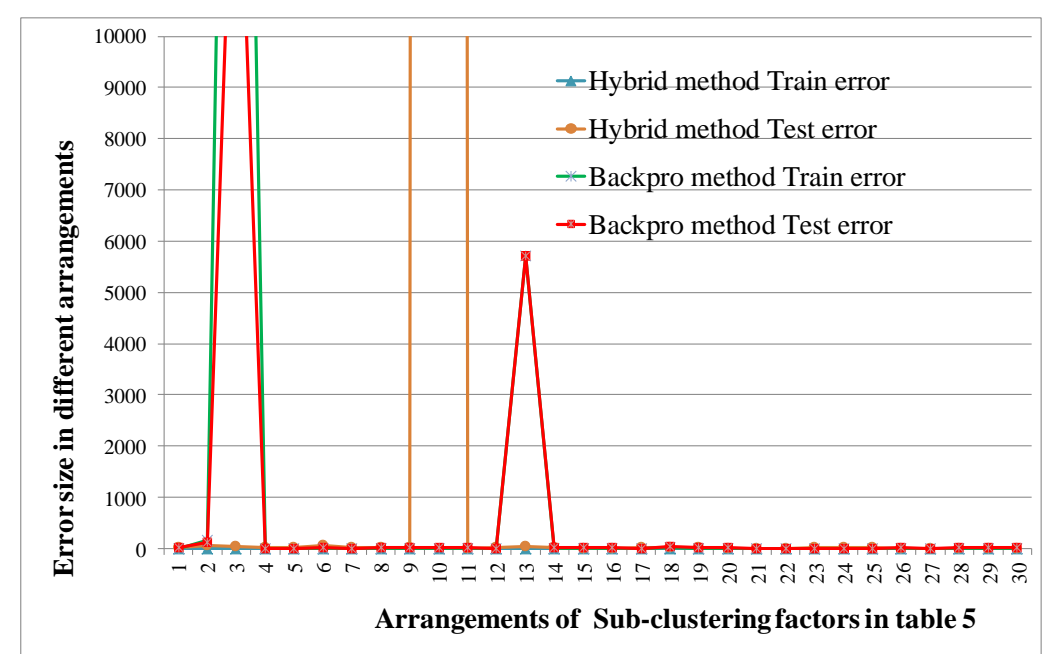

Fig. 9: Comparing the error size of training and testing in different combinations of factors in Sub-Clustering method

\section{REFERENCES}

[1] ACI-363-10, Report on High-Strength Concrete, Reported by ACI Committee 363, 1st printing, March 2010

[2] Ghosh S.K. (2004), High strength concrete in U.S codes and standards, XIV Congreso Nacional de Ingeniería Estructural, Acapulco, Gro

[3] ACI 211.4R-08, (2008), Guide for Selecting Proportions for High-Strength Concrete Using Portland Cement and Other Cementitious Materials", Reported by ACI Committee 211

[4] S. Jassar, Z. Liao, Ashrae, L. Zhao (2009), Impact of Data Quality on Predictive Accuracy of ANFIS based Soft Sensor Models, Proceedings of the World Congress on Engineering and Computer Science 2009 Vol II WCECS 2009, October 20-22, San Francisco, USA

[5] Giaccio G. and Zerbino R. (1998), Failure Mechanism of Concrete, Advanced Cement Based Materials, Volume 7(2), March 1998, pp. 41-48

[6] Kim Jin-Kuen and Han Sang-Hun (1999), Mechanical Properties of Self-Flowing Concrete, ACI Special Publication, vol. 172 pp. 637-652

[7] Shannag M.J. (2000), High strength concrete containing natural pozzolan and silica fume, Cement \& Concrete Composites, vol. 22, pp.399-406

[8] Ajdukiewicz A. and Kliszczewicz A.(2002), Influence of recycled aggregates on mechanical properties of HS/HPC, Cement \& Concrete Composites 24, pp.269279

[9] Kim J. Keun; Lee Y., Yi, S. Tae (2004), Fracture characteristics of concrete at early ages, Cement and Concrete Research vol. 34 issue 3. pp. 507-519

[10] Bissonnette B., Pigeon M. and Vaysburd Alexander M. (2007), Tensile Creep of Concrete: Study of Its Sensitivity to Basic Parameters, ACI Materials Journal, V. 104, No. 4, July-August

[11] Almeida Filho F. M., Barragan B. E., Casas J. R., and Eldebs A. L. H. C. (2008), Variability of the bond and mechanical properties of self-compacting concrete,
IBRACON structures and materials journal, Volume 1, Number 1,pp. 31-57

[12] Angel Perez J. Pablo (2008), Effect of slag cement on drying shrinkage of concrete, thesis submitted in conformity with the requirements for the degree of Master of Applied Science, September 2008, University of Toronto.

[13] Yin, J., Chi, Y., Gong, S., and Zou, W. (2010), Research and Application of Recycled Aggregate Concrete, Paving Materials and Pavement Analysis, GeoShanghai 2010 International Conference, Shanghai, Chinapp. June 3-5, pp:162-168, 10.1061/41104(377)19

[14] Ng K. M., Tam C. M. and Tam V. W. Y. (2010), Studying the production process and mechanical properties of reactive powder concrete: a Hong Kong study, Magazine of Concrete Research, Volume 62, Issue 9, pp. $647-654$

[15] Ozbay E., Gesoglu M., Guneyisi E. (2011), Transport properties based multi-objective mix proportioning optimization of high performance concretes, Materials and Structures 44:139-154

[16] Parra C., Valcuende M., Gomez F. (2011), Splitting tensile strength and modulus of elasticity of selfcompacting concrete, Construction and Building Materials 25, 201-207

[17] Das D., Chatterjee A. (2012), A comparison of hardened properties of fly-ash-based self-compacting concrete and normally compacted concrete under different curing conditions, Magazine of Concrete Research, Volume 64, Issue 2 Volume 64, Issue 2, pages 129 - 141

[18] Ranaivomanana N., Multon S., Turatsinze A. (2013), Tensile, compressive and flexural basic creep of concrete at different stress levels, Cement and Concrete Research $521-10$

[19] Joelianto E., Rahmat B. (2008), Adaptive Neuro Fuzzy Inference System (ANFIS) with Error Backpropagation Algorithm using Mapping Function, International journal of artificial intelligence, Volume 1, Number A08

[20] Ozel C., (2011), prediction of compressive strength of concrete from volume ratio and Bingham parameters 
using adaptive neuro-fuzzy inference system(Anfis) and data mining, Int. J. Physical sciences, Vol. 6(31), pp.7078-7094

[21] Sadrmomtazi, J. Sobhani, M.A. Mirgozar (2013),Modeling compressive strength of EPS lightweight concrete using regression, neural network and ANFIS, Construction and Building Materials 42, pp. 205-216

[22] Chai Y., Jia L. and Zhang Z. (2009), Mamdani Model based Adaptive Neural Fuzzy Inference System and its Application, International Journal of Information and Mathematical Sciences, 5:1

[23] Kaur A., Kaur Amrit (2012), Comparison of MamdaniType and Sugeno-Type Fuzzy Inference Systems for Air Conditioning System, International Journal of Soft Computing and Engineering (IJSCE), Volume-2, Issue-2

[24] Naderloo L., Alimardani R., Omid M., Sarmadian F., Javadikia P., Torabi M. Y., Alimardani F. (2012), Application of ANFIS to predict crop yield based on different energy inputs, Measurement 45, 1406-1413
[25] Takagi T. and Sugeno M. (1985), Fuzzy identification of systems and its applications to modeling and control, IEEE Trans. Syst., Man, Cybern, 15:116-132

[26] Duch W. (2004), Uncertainty of data, fuzzy membership functions, and multi-layer perceptrons, IEEE Transaction on neural networks, vol. XX, No. YY, 2004-1

[27] Neshat M., Adeli A., Masoumi A., sargolzae M., (2011), A Comparative Study on ANFIS and Fuzzy Expert System Models for Concrete Mix Design, IJCSI International Journal of Computer Science Issues, Vol. 8, Issue 3, No. 2

[28] Rezazadeh shirdar M., Nilashi M., Bagherifard K., Ibrahim O., Izman S., Moradifard H., Jamshidi N., Mehdi Barisamy (2011),Application of ANFIS system in prediction of machining parameter, Journal of Theoretical and Applied Information Technology, Vol 33 No.1

[29] Wei M., Bai B., Sung A. H., Liu Q., Wang J., Cather M. E. (2007), Predicting injection profiles using ANFIS, Information Sciences 177,pp. 4445-4461 